\title{
Valproic acid enhances the viability of random pattern skin flaps: involvement of enhancing angiogenesis and inhibiting oxidative stress and apoptosis
}

This article was published in the following Dove Press journal:

Drug Design, Development and Therapy

\author{
Hongqiang $\mathrm{Wu}^{1-3, *}$ \\ Jian Ding ${ }^{1-3, *}$ \\ Lei Wang ${ }^{3}$ \\ Jinti $\operatorname{Lin}^{1-3}$ \\ Shihen $\mathrm{Li}^{1-3}$ \\ Guangheng Xiang ${ }^{1-3}$ \\ Liangfu Jiang ${ }^{1-3}$ \\ Huazi $\mathrm{Xu}^{1-3}$ \\ Weiyang Gao ${ }^{1-3}$ \\ Kailiang Zhou ${ }^{1-3}$ \\ 'Department of Orthopaedics, The \\ Second Affiliated Hospital and Yuying \\ Children's Hospital of Wenzhou \\ Medical University, Wenzhou \\ 325027, China; ${ }^{2}$ Zhejiang Provincial \\ Key Laboratory of Orthopaedics, \\ Wenzhou 325027, China; ${ }^{3}$ The Second \\ Clinical Medical College of Wenzhou \\ Medical University, Wenzhou 325027, \\ China \\ *These authors contributed equally \\ to this work
}

\begin{abstract}
Background: Random skin flaps are commonly applied during plastic surgery, but distal flap necrosis limits their clinical applications. Valproic acid (VPA), a histone deacetylase inhibitor and a traditional antiepileptic agent, may promote flap survival.

Materials and methods: Sprague-Dawley rats were randomly divided into VPA-treated and control groups. All rats received VPA or saline by intraperitoneal injections once daily for 7 days after the modified McFarlane flap model was established. On postoperative day 7, flap survival, laser Doppler blood flow, and water content were examined for flap viability, hematoxylin and eosin staining (H\&E), immunohistochemistry (IHC), and Western blot analysis, and the status of angiogenesis, apoptosis, and oxidative stress were detected in the ischemic flaps.

Results: VPA increased the survival area, blood flow, and number of microvessels in skin flaps on postoperative day 7 and reduced edema. VPA promoted angiogenesis by enhancing vascular endothelial growth factor (VEGF) mRNA transcription and upregulating VEGF and cadherin 5 expression, inhibited apoptosis via reduction of caspase 3 cleavage, and relieved oxidative stress by increasing superoxide dismutase (SOD) and glutathione (GSH) levels and reducing the malondialdehyde (MDA) level.
\end{abstract}

Conclusion: VPA promoted random skin flap survival by enhancing angiogenesis and inhibiting oxidative stress and apoptosis.

Keywords: valproic acid, random skin flap, angiogenesis, oxidative stress, apoptosis

\section{Introduction}

Random-pattern skin flaps are commonly used to cover superficial skin defects during plastic surgery. ${ }^{1}$ However, the length-to-width ratio of the flap cannot exceed $2: 1$; otherwise, the distal areas are vulnerable to necrosis. ${ }^{2}$ Previous studies suggested that inadequate blood flow, ischemia-reperfusion (I/R) injury, oxidative stress, and apoptosis contributed to distal flap necrosis., ${ }^{3,4}$ Random skin flaps exhibit significant ischemia when blood flow is inadequate. Vascular regeneration commences at the flap pedicle; the blood supply then gradually increases. Flap ischemia and blood restoration trigger I/R injury. ${ }^{5}$ Oxidative stress plays a key role in the progression of such injury, which can cause extensive cell apoptosis if untreated. ${ }^{6}$ Thus, pharmacological agents promoting angiogenesis and alleviating oxidative stress and apoptosis would be expected to improve random skin flap survival.

Valproic acid (VPA, 2-propylpentanoic acid), a histone deacetylase inhibitor, is traditionally used to treat epilepsy and bipolar disorder. ${ }^{7}$ VPA is also used to treat

\footnotetext{
Correspondence: Weiyang Gao: Kailiang Zhou

Department of Orthopaedics, The Second Affiliated Hospital and Yuying Children's Hospital of Wenzhou Medical University, 109 West Xueyuan Road, Wenzhou 325027, Zhejiang, China Tel +86 57788002812 ; +86577880028I5 Email weiyanggaoi@I26.com; zhoukailiang@wmu.edu.cn
} 
neurological and/or neurodegenerative diseases, including stroke, traumatic brain injury, and Huntington's disease, affording neuroprotection, inhibiting oxidative stress, and promoting neurovascular remodeling. ${ }^{8}$ It is safe and effective, enhancing angiogenesis and promoting functional recovery by inhibiting histone deacetylase and upregulating hypoxiainducible factor (HIF)- $1 \alpha$ and the downstream pro-angiogenic factors vascular endothelial growth factor (VEGF) and matrix metalloproteinase (MMP)-2/9 in a rat model of ischemic stroke. ${ }^{9,10}$ Moreover, chronic lithium/VPA treatment exerted neuroprotective effects, inhibiting excitotoxicity and glutamate-induced cell death by reducing oxidative damage. ${ }^{11}$ One study found that VPA protected endothelial cells from stress-induced apoptosis by inducing endothelial cell ERK $1 / 2$ phosphorylation. ${ }^{12}$ In addition, VPA exerted a beneficial effect in an experimental Adriamycin ${ }^{\circledR}$-induced model of nephropathy through reducing glomerular apoptosis. ${ }^{13}$

However, the effect of VPA on random-pattern skin flaps remains poorly understood. Here, we explored whether VPA promoted the survival of random rat skin flaps through enhancing angiogenesis and inhibiting oxidative stress and apoptosis.

\section{Materials and methods Ethics statement}

All animal experiments were approved by the Animal Research Committee of Wenzhou Medical University (wydw2017-0022) and accorded with the Guide for the Care and Use of Laboratory Animals of China National Institutes of Health.

\section{Reagents and antibodies}

VPA $\left(\mathrm{C}_{8} \mathrm{H}_{16} \mathrm{O}_{2}\right.$, purity $\left.>99 \%\right)$ was purchased from TCI chemicals (Shanghai, China). Hematoxylin and eosin (H\&E) staining kit and pentobarbital sodium were purchased from Solarbio Science \& Technology (Beijing, China). The primary antibody against CD34 was obtained from Abcam (Cambridge, UK). Primary antibodies against VEGF, superoxide dismutase (SOD), and $\beta$-actin were obtained from Beijing Bioss Biotechnology (Beijing, China). SOD, glutathione (GSH), and malondialdehyde (MDA) assay kits were purchased from the Nanjing Jiancheng Bioengineering Institute (Jiangsu, China). Primary antibody against cleaved caspase 3 (CASP3) was purchased from Cell Signaling Technology (Beverly, MA, USA). A VEGF mRNA in situ hybridization kit and the primary antibody against cadherin 5 were purchased from Boster Biological Technology (Wuhan, China). Horseradish peroxidase (HRP)-conjugated goat-anti-rabbit
IgG was obtained from Boyun Biotechnology (Nanjing, China). A bicinchoninic acid test kit was purchased from Beijing ComWin Biotech Company (Beijing, China). An electrochemiluminescence-plus immunodetection kit was obtained from Merck Millipore (Billerica, MA, USA).

\section{Animals and groups}

Sixty male Sprague-Dawley rats were purchased from Experimental Animal Center of Wenzhou Medical University (License no SCXK 2005-0019). All rats were randomly divided into two groups: a control group ( $\mathrm{n}=30$, control group) and a VPA group ( $n=30$, VPA group). Each rat was housed separately in a cage and allowed free access to food and water in an environmentally controlled animal house.

\section{The modified McFarlane flap model}

After anesthesia with $2 \%(\mathrm{w} / \mathrm{v})$ pentobarbital sodium (40 $\mathrm{mg} / \mathrm{kg}$ intraperitoneally) for each rat, the dorsal fur was removed with an electric shaver and depilatory cream. Then caudal-based $3 \times 9 \mathrm{~cm}$ areas were outlined in the rat dorsum and elevated beneath the panniculus carnosus as previously reported. ${ }^{2}$ All known vessels were completely sectioned, and each flap was immediately sutured with 4-0 non-absorbable suture in the original position. Each flap was separated into three equal zones: proximal (area I), intermediate (area II), and distal (area III) zones.

\section{Drug administration}

Each rat in the VPA group received $200 \mathrm{mg} / \mathrm{kg}$ VPA by intraperitoneal injections after the modified McFarlane flap model was established, 12 hours later, and then once daily until euthanasia. Rats in the control group received equal volumes of saline in accordance with the principles of administration in the VPA group.

\section{Flap survival evaluation}

After establishment of the flap model, appearance, color, texture, and hair condition of flaps were noted for 7 consecutive days. On postoperative days 3 and 7, high-quality photographs of the random flap were acquired to evaluate the flap viability via grid counting. Percentages of viable area were calculated as follows: survival area/total area of flap $\times 100 \%$.

\section{Tissue edema assessment}

Tissue edema was reflected by the water content of flaps. On postoperative day 7 , the flaps were weighed, then dehydrated in an autoclave at $50^{\circ} \mathrm{C}$ and weighed again until the weight was stabilized for 2 days. Percentage of water 
content was determined as: ([wet weight - dry weight]/wet weight) $\times 100 \%$.

\section{Laser Doppler blood flow imaging}

On postoperative day 7, a laser Doppler instrument (Moor Instruments, Axminster, UK) was used to measure blood flow of flap in a warm and quiet environment under anesthesia. Perfusion units were used as indicators of blood flow. The blood flow of skin flap was quantified with moor LDI Review software (ver.6.1; Moor Instruments).

\section{H\&E staining}

On postoperative day 7 , specimens $(n=6,0.5 \times 0.5 \mathrm{~cm})$ of central tissue from each flap area II were obtained and executed after sacrifice. Then the samples were first fixed in 4\% paraformaldehyde for 24 hours and embedded in paraffin wax for transverse sectioning. Sections with $4 \mu \mathrm{m}$ of thickness were prepared and mounted on poly-L-lysine-coated slides for $\mathrm{H} \& \mathrm{E}$ staining. The number of microvessels per unit area $\left(/ \mathrm{mm}^{2}\right)$ was calculated under a light microscope $(\times 200$ magnification; Olympus Corporation, Tokyo, Japan), which indicated mean microvascular density. Six random fields of three random sections from each tissue specimen were measured.

\section{Immunohistochemistry (IHC)}

Six sections of the central part of area II in each group were dewaxed in xylene and rehydrated in graded ethanol baths on day 7. After washing, the sections were blocked with $3 \%$ (v/v) $\mathrm{H}_{2} \mathrm{O}_{2}$ and placed in $10.2 \mathrm{mM}$ sodium citrate buffer for 30 minutes at $95^{\circ} \mathrm{C}$. Then the sections were blocked with $10 \%(\mathrm{w} / \mathrm{v})$ bovine serum albumin and phosphate buffered saline for 10 minutes and incubated with primary antibody against CD34 (1:100), VEGF (1:200), cadherin 5 (1:100), CASP3 (1:200), and SOD (1:100) overnight at $4^{\circ} \mathrm{C}$. Finally, the sections were incubated with HRP-conjugated secondary antibody $(1: 1,000)$, stained with 3,3'-diaminobenzidine, and counterstained with hematoxylin. Tissue sections were imaged at $\times 200$ magnification under a light microscope (Olympus Corporation). The images were analyzed with Image-Pro Plus software (Media Cybernetics, Rockville, MD, USA) for integral absorbance quantitation of VEGF-, CASP3-, SOD-, and CD34-positive blood vessel counting. Six random fields of three random sections from each tissue sample were counted.

\section{In situ hybridization}

An in situ hybridization kit was used to examine VEGF mRNA in the flap tissue. The probes for VEGF mRNA were 5'-GCTCT
ACCTCCAC CA TGCCA AGTGG TCCCA-3', 5'-GACCC TGGTGGACATCTTCCAGGAGTACCC-3', and 5'-GCAGC TTGAG TTAAA CGAAC GTACT TGCAG-3'. In situ hybridization was performed as described previously. ${ }^{14}$ After staining with 3,3'-diaminobenzidine, the sections were counterstained with hematoxylin and sealed. Under a light microscope ( $\times 400$ magnification; Olympus Corporation), six random fields of three random sections from each flap tissue sample were counted. Integral absorbance quantitation of VEGF transcription was counted using Image-Pro Plus software.

\section{Western blot analysis}

On postoperative day 7 , specimens $(n=6,0.5 \times 0.5 \mathrm{~cm})$ from the center of area II were dissected and stored at $-80^{\circ} \mathrm{C}$ before Western blot analysis. Proteins were extracted from flap samples with a lysis buffer and calculated by the BCA assay. They were separated by $10 \%$ polyacrylamide gel electrophoresis and transferred to polyvinylidene fluoride (PVDF) membranes (Merck Millipore). After blocking with 5\% (w/v) non-fat milk for 2 hours at room temperature, the membranes were incubated with the following primary antibodies at $4^{\circ} \mathrm{C}$ overnight: VEGF (1:1,000), SOD (1:1,000), cleaved-CASP3 (1:1,000), and $\beta$-actin $(1: 1,000)$, and then incubated with goat-anti-rabbit secondary antibody for 1.5 hours at room temperature. The immunoreactive proteins were visualized using the ECL Plus Reagent Kit (Merck Millipore, Billerica, MA, USA). Finally, the band intensity was quantified using Gel Image system ver.4.00 (Tanon, Shanghai, China).

\section{SOD activity, GSH level, and MDA content}

On postoperative day 7 , tissue specimens from the center of area II $\left(\mathrm{n}=6,0.5 \times 0.5 \mathrm{~cm}^{2}\right)$ were obtained and used to evaluate SOD activity, GSH level, and MDA content using commercial kits as described previously. ${ }^{15}$ SOD activity, GSH level, and MDA content were determined using the xanthine oxidase method, 5,5'-dithiobis method, and thiobarbituric acid test, respectively.

\section{Statistical analyses}

All data were expressed as mean \pm standard error ofmean (SEM). Statistical analyses were conducted using SPSS software version 19.0 (IBM Corporation, Armonk, NY, USA). The data of the two groups were compared using an independentsample $t$-test. $P<0.05$ indicates statistical significance.

\section{Consent for publication}

We declare that our institutes are aware of the work and declare consent for publication of the manuscript. 


\section{Results}

\section{VPA affected the viability of random skin flaps}

On day 3 after the surgery, the flaps in both the groups were pale and swollen in area III and the distal portion of the flaps became dark (Figure 1A). The VPA group showed a greater survival area relative to the control group on day 3 $(82.33 \% \pm 2.82 \%$ and $64.90 \% \pm 4.83 \%$, respectively; $P=0.011$; Figure 1B). By day 7 , each group exhibited survival in area I, whereas area III had become darker along with scabbing and hardening, indicating necrosis (Figure 1A). The mean survival area of the group treated with VPA was significantly larger than that of the control group on day 7 $(64.34 \% \pm 4.30 \%$ and $44.02 \% \pm 2.61 \%$, respectively; $P=0.002$; Figure 1B). Furthermore, the signal intensity of blood flow within the flap detected by laser Doppler instrument was much larger in the VPA group than that in the control group (541.93 \pm 26.00 and 418.92 \pm 31.23 PU, respectively; $P=0.013$; Figure $1 \mathrm{C}$ and D). Finally, the distal part of the flaps in the control group was significantly edematous with subcutaneous venous blood congestion (Figure 1E). The water content of tissue was lower in the VPA group than that in the control group $(44.39 \% \pm 3.54 \%$ and $59.40 \% \pm 4.21 \%$, respectively; $P=0.021$; Figure 1F).
A
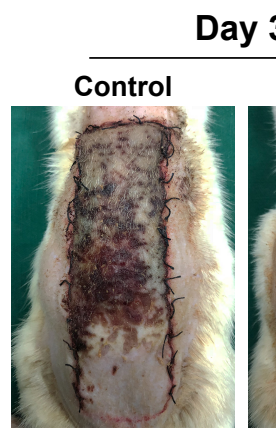

Day 7

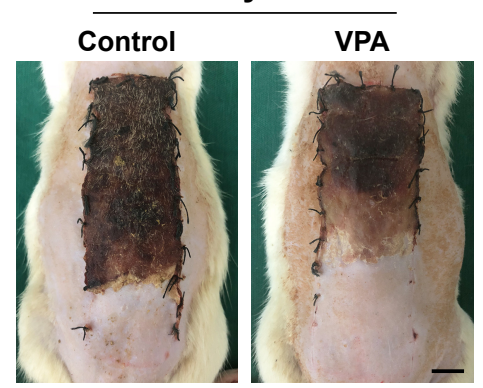

B

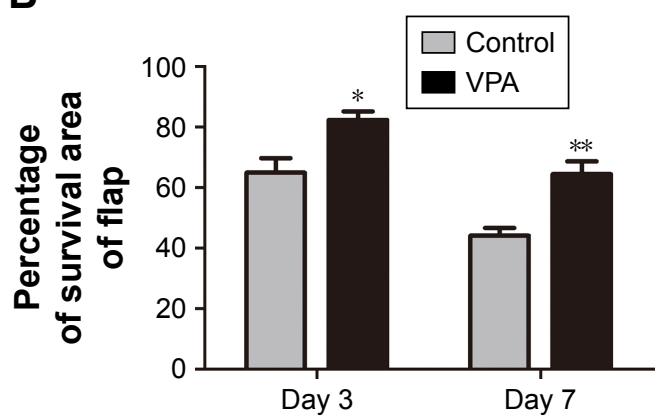

C

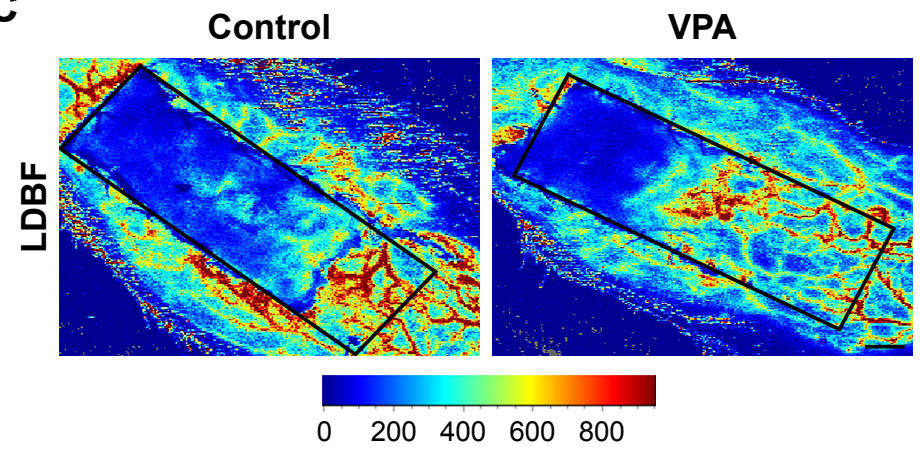

E

Control

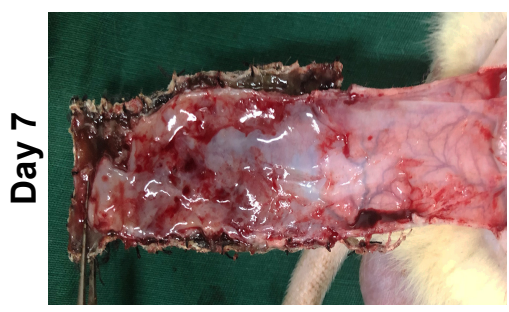

VPA

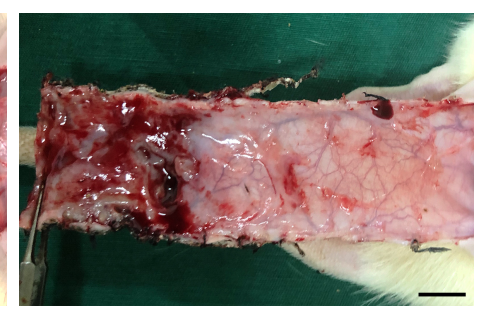

D

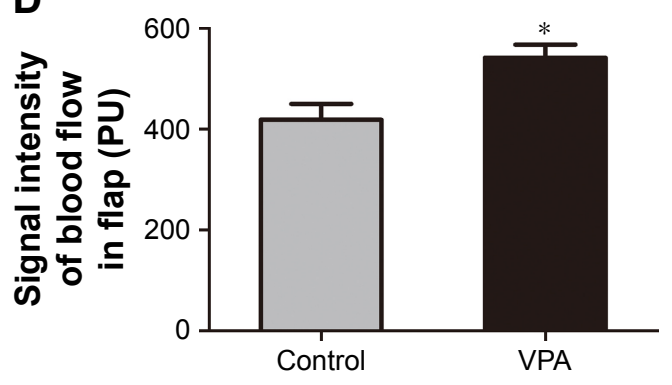

$\mathbf{F}$

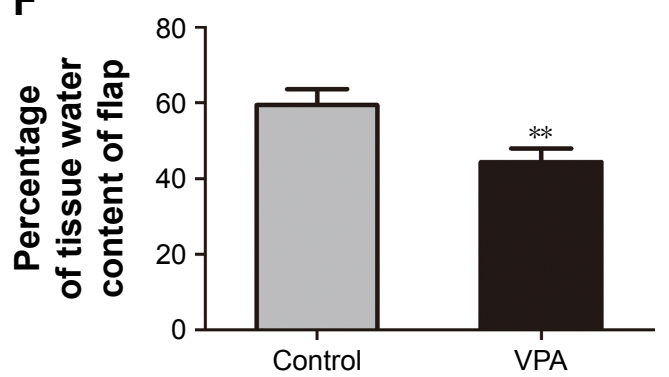

Figure I Effects of VPA on the viability of random skin flaps.

Notes: (A) Digital photographs show the postoperative flaps on days 3 and 7 (scale bar, $1 \mathrm{~cm}$ ). (B) Histogram of percentages of survival area on days 3 and 7 . (C) Full-field laser Doppler blood flow images of flaps on day 7 (scale bar, I cm). (D) Histogram of blood flow on day 7. (E) Digital photographs show the tissue edema of postoperative flaps of each group on day 7 (scale bar, I cm). (F) Histogram of percentage of water content in the tissue. Values are expressed as mean \pm SEM, $n=6$ per group. $* P<0.05$ and $* * P<0.01$ vs control group.

Abbreviations: LDBF, laser Doppler blood flow; SEM, standard error of mean; VPA, valproic acid. 


\section{VPA increased the number of microvessel in random skin flaps}

On day 7 , specimens of central tissue from each flap in area II were stained with H\&E and IHC. The VPA group generated a greater number of microvessels relative to the control group (Figure 2A). The mean vessel density of area II was greater in the VPA group $\left(262.10 \pm 21.34 / \mathrm{mm}^{2}\right)$ than in the control group $\left(128.27 \pm 15.36 / \mathrm{mm}^{2} ; P<0.001\right.$; Figure 2B). In addition, the number of CD34-positive vessels was greater in the VPA group than in the control group (293.18 $\pm 39.28 /$ and $145.69 \pm 14.35 / \mathrm{mm}^{2}$, respectively; $P=0.005$; Figure $2 \mathrm{C}$ and $\mathrm{D}$ ).

\section{VPA promoted angiogenesis in random skin flaps}

On postoperative day 7, in situ hybridization for VEGF mRNA in area II of all flaps was performed to evaluate the level of angiogenesis. As shown in Figure 3A, more VEGF mRNA was synthesized in the vascular structures of the cutis and dermis in the VPA group compared with the control group. There was a statistical difference in VEGF mRNA transcription between the VPA group and the control group ( $P=0.016$; Figure $3 \mathrm{~B}$ ). We also detected VEGF and cadherin 5 to confirm the level of angiogenesis. VEGF was detected in vessels and stromal cells in area II and at a higher level in the VPA group than in the control group $(P=0.002$; Figure $3 \mathrm{C}$ and $\mathrm{D}$ ), which was the same as cadherin 5 expression in vessels and stromal cells ( $P=0.011$; Figure $3 \mathrm{E}$ and $\mathrm{F})$. Western blot analysis revealed that VEGF was significantly upregulated in the VPA group compared with the control group $(P<0.001$; Figure $3 \mathrm{G}$ and $\mathrm{H})$.

\section{VPA inhibited oxidative stress in random skin flaps}

To examine the effects of VPA on oxidative stress in random skin flaps, SOD levels were analyzed by Western blot analysis and IHC. IHC and integral absorbance analyses showed higher levels of SOD in the dermis of the VPA group, relative to the control group $(P=0.001$; Figure $4 \mathrm{~A}$ and $\mathrm{B})$, which was in accordance with that seen in Western blot analysis $(P<0.001$; Figure $4 \mathrm{C}$ and D). SOD activity, GSH level, and MDA content were also measured using kits. The SOD activity was greater in the VPA group than that in the control group $(56.42 \pm 4.37$ and $38.54 \pm 5.41 \mu / \mathrm{mg} /$ protein, respectively; $P=0.028$; Figure 4E). Moreover, GSH level was increased in the VPA group $(3.34 \pm 0.40 \mathrm{nmol} / \mathrm{mg} /$ protein $)$ compared with the control group $(2.25 \pm 0.23 \mathrm{nmol} / \mathrm{mg} /$ protein; $P=0.040$; Figure $4 \mathrm{~F}$ ), whereas MDA content was decreased $(40.66 \pm 4.12$ and $63.50 \pm 5.54 \mathrm{nmol} / \mathrm{mg} /$ protein $P=0.008$; Figure 4G).
A

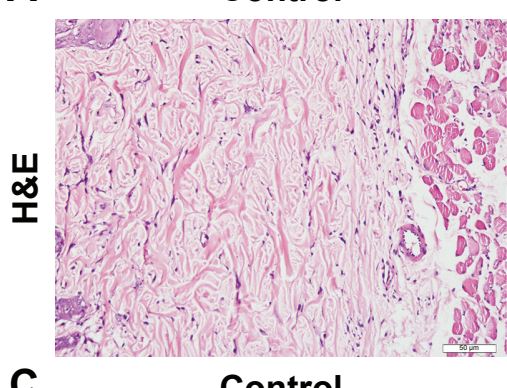

C

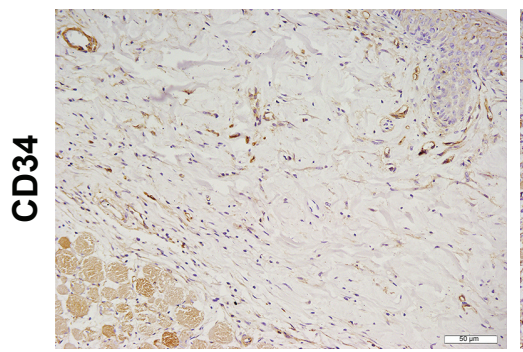

VPA

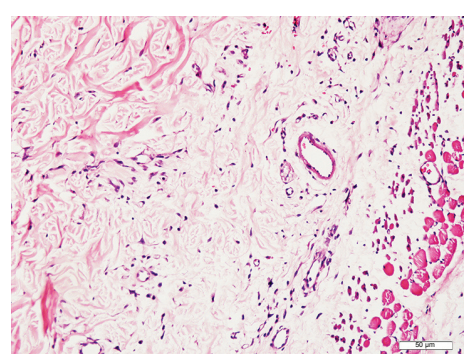

VPA

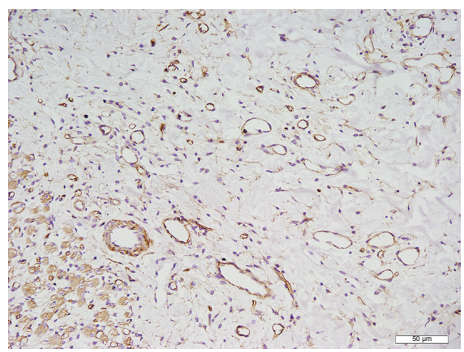

B

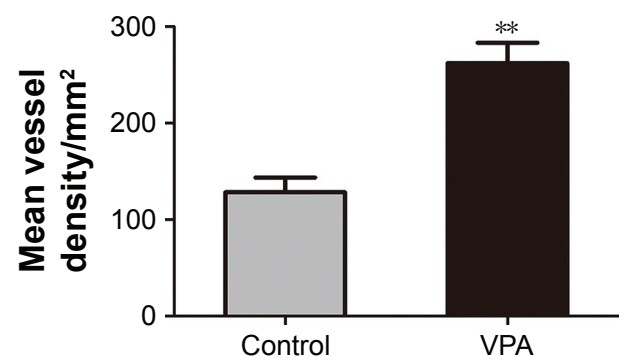

D

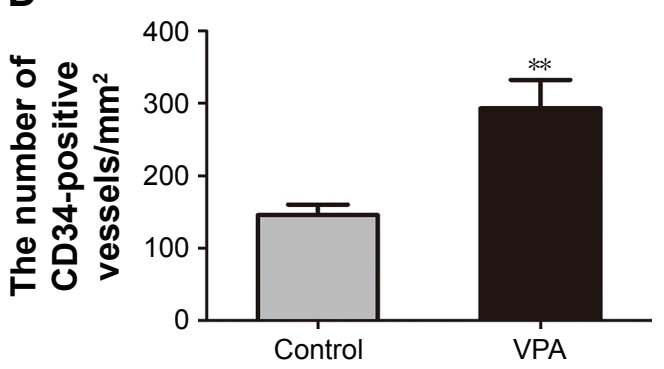

Figure 2 VPA increased the number of microvessels in random skin flaps.

Notes: (A) Neovascularization in control and VPA groups by H\&E staining (original magnification $\times 200$ ). (B) Histogram of MVDs on day 7. (C) CD34-positive vessels as assessed by IHC (original magnification $\times 200$ ). (D) Histogram of the number of CD34-positive vessels on day 7 . Values are expressed as mean \pm SEM, $n=6$ per group. $* * P<0.0$ I, vs control group.

Abbreviations: H\&E, hematoxylin and eosin; IHC, immunohistochemistry; MVD, microvascular density; SEM, standard error of mean; VPA, valproic acid. 

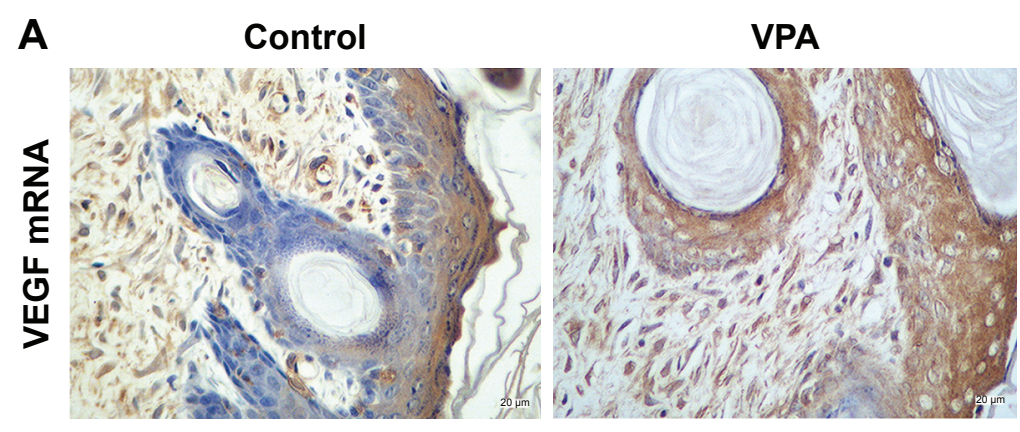

C

Control
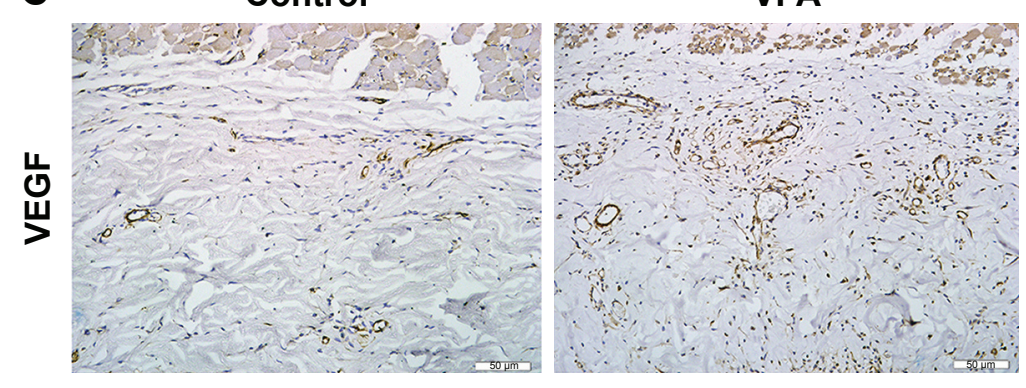

E

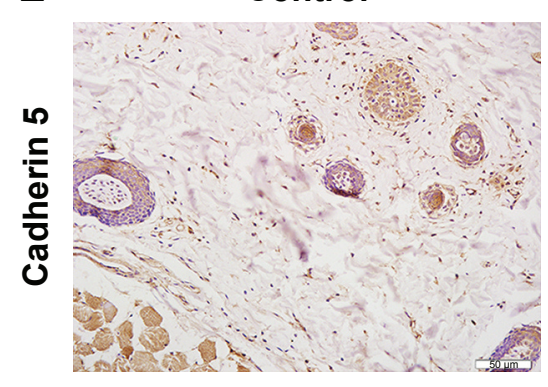

Control

G

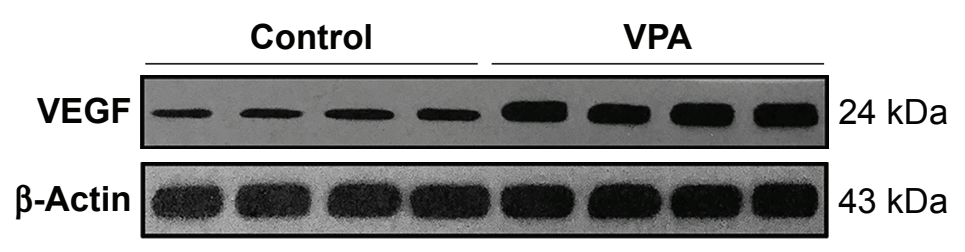

B

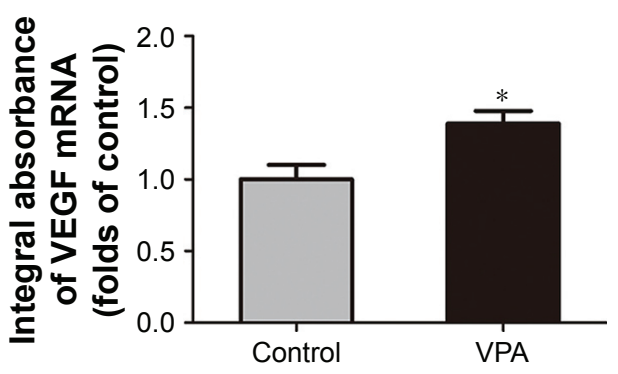

D

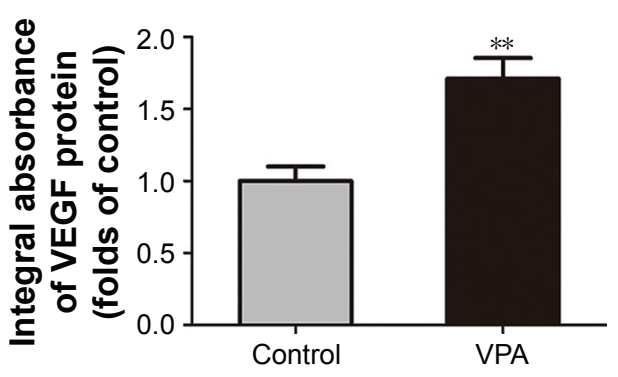

$\mathbf{F}$
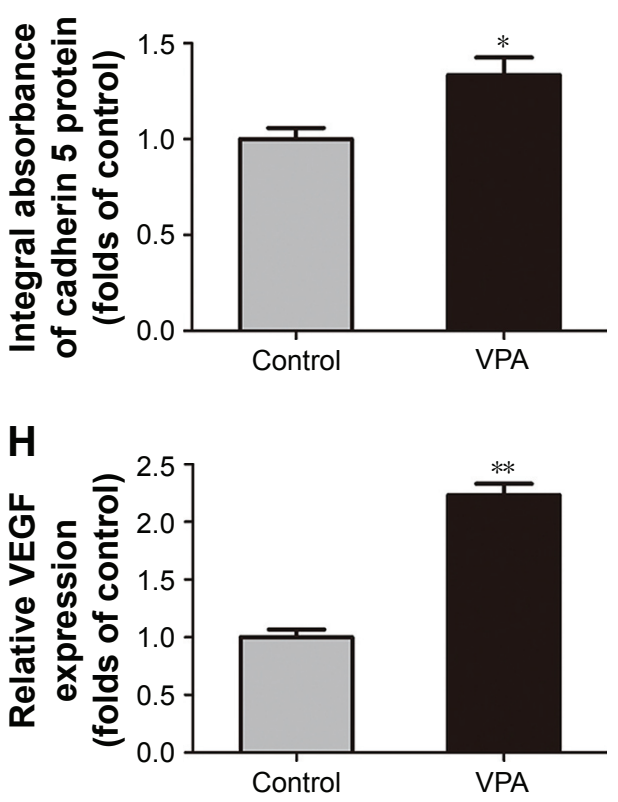

Figure 3 VPA promoted angiogenesis in random skin flaps.

Notes: (A) In situ hybridization of VEGF mRNA on day 7 (original magnification $\times 400$ ). (B) The optical density values of VEGF mRNA. (C and E) VEGF and cadherin 5 expressions in each group as assessed by IHC (original magnification $\times 200$ ). (D and F) The optical density values of VEGF and cadherin 5 . (G) Protein expression of VEGF in each group, as assessed by Western blot analysis. The gels have been run under the same experimental conditions, and cropped blots are used here. (H) Densitometry results of VEGF protein expression in the two groups. Values are expressed as mean \pm SEM, $n=6$ per group. $* P<0.05$ and $* * P<0.0$ I, vs control group.

Abbreviations: IHC, immunohistochemistry; SEM, standard error of mean; VEGF, vascular endothelial growth factor; VPA, valproic acid.

\section{VPA reduced apoptosis in random skin flaps}

Cleaved CASP 3 expression was used to evaluate the level of apoptosis. IHC and integral absorbance analyses of the dermis demonstrated that cleaved CASP3 levels were remarkably decreased in comparison with the control group ( $P=0.028$; Figure 5A and $\mathrm{C}$ ). In addition, Western blot analysis also revealed that the expression of cleaved CASP3 was significantly downregulated in the 
A

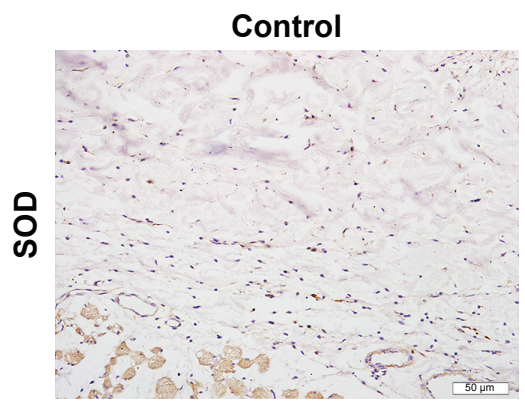

C

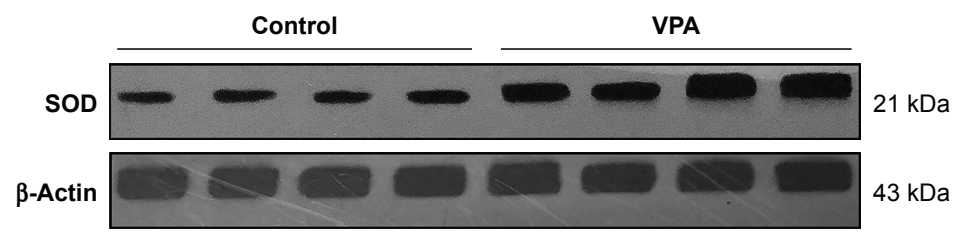

E

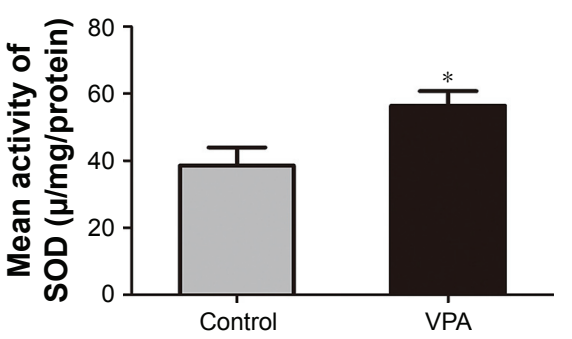

VPA

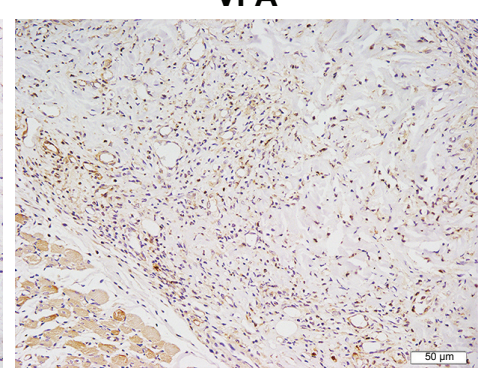

$F$

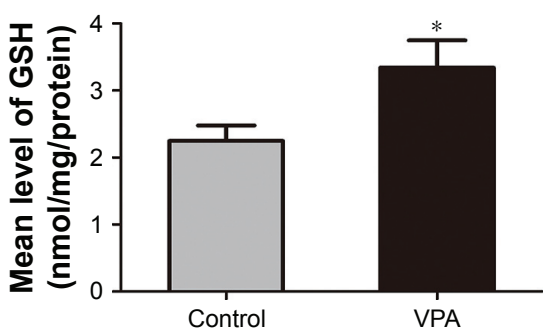

G
B

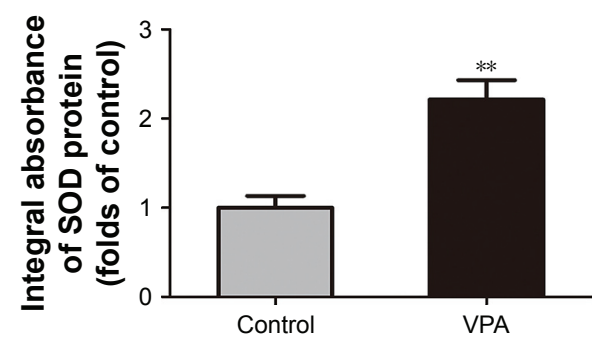

D
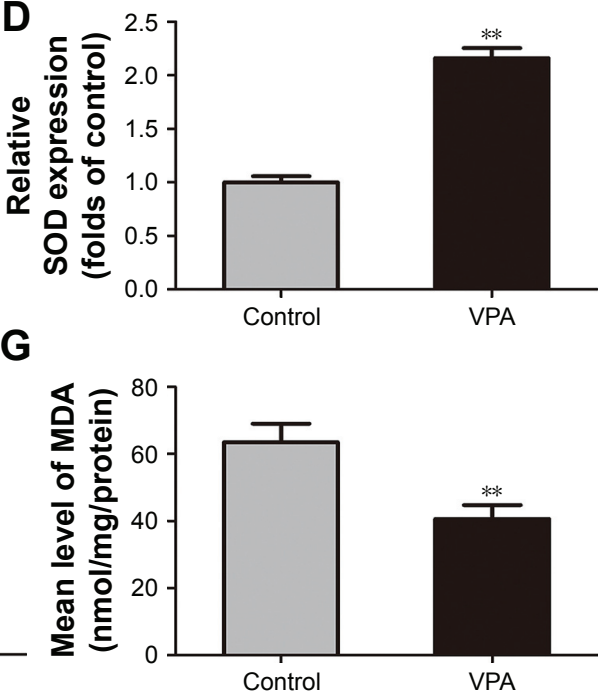

Figure 4 VPA inhibited oxidative stress in random skin flaps.

Notes: (A) SOD expression in each group as assessed by IHC (original magnification $\times 200$ ). (B) The optical density values of SOD. (C) Protein expression of SOD in each group as assessed by Western blot analysis. The gels have been run under the same experimental conditions, and cropped blots are used here. (D) Densitometry result of SOD protein expression in the two groups. (E-G) The levels of SOD, GSH, and MDA in random skin flaps were determined using the xanthine oxidase method, 5,5'-dithiobis method, and thiobarbituric acid test, respectively. Values are expressed as mean $\pm S E M, n=6$ per group. $* P<0.05$ and $* * P<0.01$, vs control group.

Abbreviations: GSH, glutathione; IHC, immunohistochemistry; MDA, malondialdehyde; SEM, standard error of mean; SOD, superoxide dismutase; VPA, valproic acid.

VPA group compared with the control group $(P=0.001$; Figure 5B and D).

\section{Discussion}

VPA is traditionally used to treat epilepsy and bipolar disorder. ${ }^{16}$ Previous studies showed that VPA might usefully treat neurological and/or neurodegenerative diseases, including stroke, traumatic brain injury, and Huntington's disease, affording neuroprotection, minimizing oxidative stress, and preventing neurovascular remodeling. ${ }^{8,21,32}$ In clinical practice, distal flap necrosis caused by $\mathrm{I} / \mathrm{R}$ injury and apoptosis limit clinical flap applications. ${ }^{17}$ No study has explored whether VPA reduces ischemia in random skin flaps. We found that VPA improved random skin flap survival by enhancing neovascularization of affected areas and inhibiting oxidative stress and apoptosis.

In several studies, VPA was proangiogenic in models of ischemic stroke and hemorrhagic shock. ${ }^{10,18}$ VPA enhances postischemic angiogenesis via upregulation of HIF- $1 \alpha$ and the downstream pro-angiogenic factors VEGF and MMP-2/9. ${ }^{10}$ However, in other studies, VPA was antiangiogenic in models of several cancers. ${ }^{19,20}$ Thus, the effect of VPA on angiogenesis remains controversial, perhaps attributable to differential regulation of key genes and proteins in various disease microenvironments. The effect of VPA on random skin flap angiogenesis remains poorly understood. In the present study, $\mathrm{H} \& \mathrm{E}$ and IHC staining for $\mathrm{CD} 34$ revealed significantly increased number of microvessels in the dermis of flaps treated with VPA. Also, LBDF imaging showed that flap blood flow increased in the VPA group. Thus, VPA promoted flap survival by increasing the number of microvessels and restoring the blood supply. Angiogenesis involves the destruction of pre-existing cell connections, followed by mitosis, endothelial cell sprouting, and maturation of new capillaries; VEGF initiates signal cell sprouting. ${ }^{22}$ In addition, cadherin 5 plays an important role in migration and positional changes of angiogenic endothelial cells. ${ }^{23} \mathrm{We}$ found that VPA upregulated VEGF mRNA and protein levels in both vessels and stromal cells. Moreover, increased expression of VEGF in dermal flaps, as demonstrated by Western blot analysis, 
A

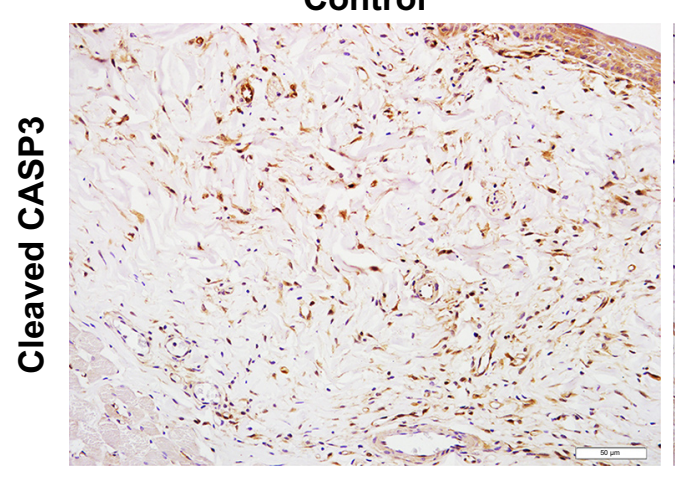

B
Control

Control
VPA

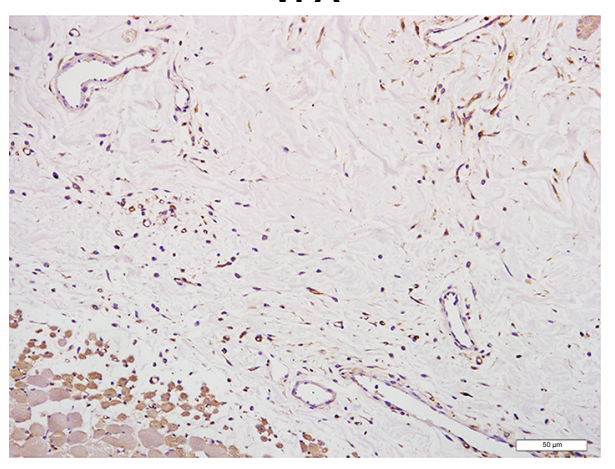

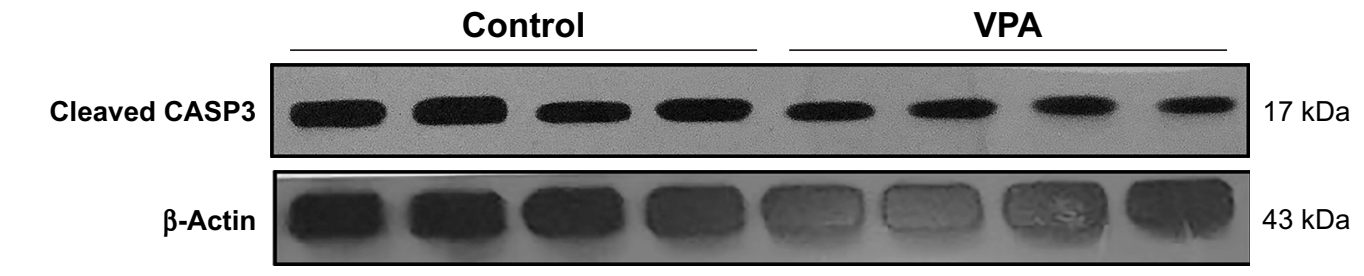

C

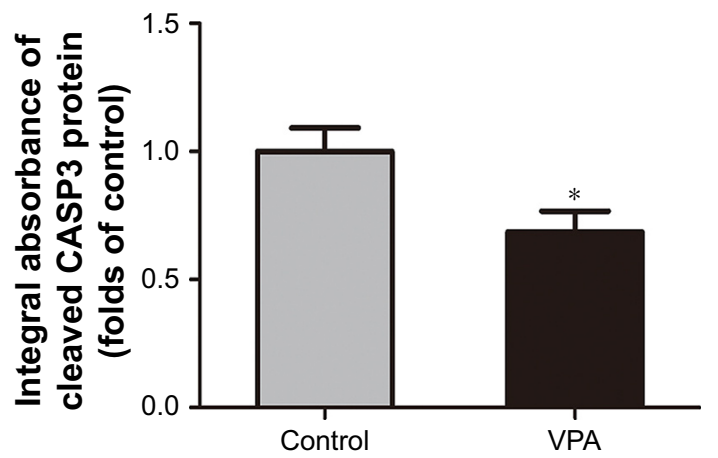

D

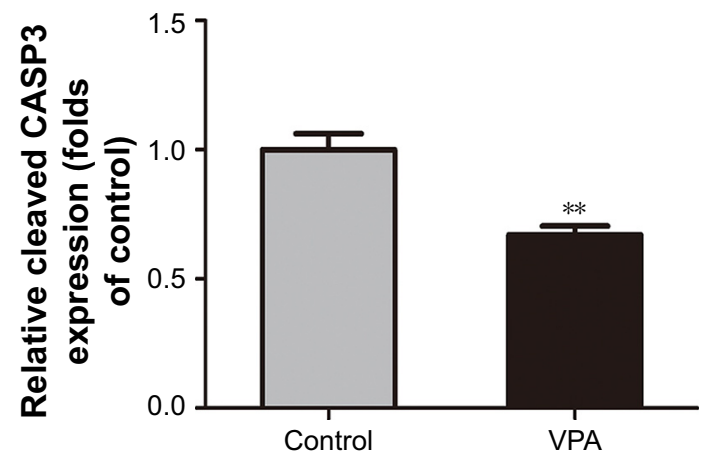

Figure 5 VPA reduced apoptosis in random skin flaps.

Notes: (A) Cleaved CASP3 expression in each group as assessed by IHC (original magnification $\times 200$ ). (B) Protein expression of cleaved CASP3 in each group as assessed by Western blot analysis. The gels have been run under the same experimental conditions, and cropped blots are used here. (C) The optical density values of cleaved CASP3. (D) Densitometry result of cleaved CASP3 protein expression in the two groups. Values are expressed as mean $\pm S E M$, $n=6$ per group. $* P<0.05$ and $* * P<0.01$, vs control group. Abbreviations: IHC, immunohistochemistry; SEM, standard error of mean; VPA, valproic acid.

was validated via IHC. We also found that VPA increased cadherin 5 expression in dermal flaps as determined by IHC. Together, these data indicated that VPA enhanced flap viability by upregulating VEGF-induced angiogenesis and cadherin 5 expression.

Previous studies suggested that I/R injury contributed to necrosis of the distal regions of random skin flaps. ${ }^{24-26}$ Prevention of I/R injury may improve random skin flap survival. $\mathrm{I} / \mathrm{R}$ injury is a complex process, involving energy metabolism, apoptosis, oxidative stress, platelet aggregation, and leukocyte/endothelium interactions. ${ }^{27,28}$ Of these processes, oxidative stress generates the highest levels of deleterious ROS and can trigger extensive cell apoptosis if untreated..$^{6,29}$ VPA significantly reduced MDA levels and increased SOD activity and GSH levels, in lipopolysaccharide-treated rats. ${ }^{30}$
SOD and GSH are believed to be essential scavengers alleviating tissue injury caused by peroxidase reactions. ${ }^{31} \mathrm{MDA}$, a toxic final product of lipid peroxidation, serves as an indicator of the severity of lipid peroxidation. ${ }^{33}$ In the present study, SOD activity and the GSH level were increased, and the MDA level was decreased, in the VPA group. Thus, VPA prevented I/R injury by alleviating oxidative stress.

Apoptosis is the primary form of cell death during skin flap I/R injury. ${ }^{34}$ Apoptosis exacerbates the distal necrosis of random skin flaps. VPA protects against apoptosis both in vitro and in vivo. ${ }^{12,13,35}$ VPA induced ERK 1/2 phosphorylation in endothelial cells, thereby protecting them against apoptosis. ${ }^{12}$ Moreover, VPA inhibited CASP3 activation and motor neuron death by reducing mitochondrial cytochrome c release and oxidative stress after spinal 
cord injury. ${ }^{36}$ In general, apoptosis is associated with an intracellular caspase cleavage cascade. ${ }^{37}$ CASP3 is one of the essential effectors of apoptosis, contributing to the proteolytic cleavage of many key proteins. ${ }^{38} \mathrm{CASP} 3$ cleavage/ activation is the convergence point of two apoptotic pathways and the final step in cell death initiation. ${ }^{37}$ Thus, we measured cleaved CASP3 levels to assess the extent of apoptosis in response to VPA treatment. The level of cleaved CASP3 was remarkably lower in the VPA than in the control group. Thus, VPA attenuated apoptosis in ischemic flaps.

However, there is no doubt that the limitations of VPA in random skin flap therapy still need to be further investigated. For example, the treatment of a single concentration of VPA was performed daily after the flap establishment; the optimal concentration postoperation administration should be identified in further study. In addition, the study would be strengthened by detecting the serum concentration of VPA at time points after the drug treatment when steady state levels are expected to be reached to confirm that physiologically relevant levels were administered. Moreover, for further translational research, application in large animal model (proof of concept as a preclinical study) will be beneficial; therefore, rabbit or pig will be used to verify the beneficial effect of VPA on the flaps survival in the future.

\section{Conclusion}

VPA improved random skin flap survival by promoting angiogenesis and inhibiting oxidative stress and apoptosis. Further experimental and clinical studies are required to confirm that VPA is of clinical utility in patients receiving random skin flaps.

\section{Acknowledgments}

This work was supported by grants from Natural Science Foundation of China (No 81601705 to Kailiang Zhou, No 81873942 to Weiyang Gao, No 81801930 to Jian Ding, No 81572227 and No 81873992 to Huazi Xu); Zhejiang Provincial Medicine and Health Technology Project (No 2017KY472 to Kailiang Zhou); and Wenzhou Science and Technology Bureau Foundation (No 2016 Y0350 to Jian Ding).

\section{Author contributions}

HW and JD wrote the manuscript text. HW, JD, LW, JL, and SL prepared figures and collected samples. GX and LJ analyzed data. WG and $\mathrm{KZ}$ designed the experiment. $\mathrm{HX}, \mathrm{WG}$, and $\mathrm{KZ}$ revised manuscript. All authors contributed to data analysis, drafting or revising the article, gave final approval of the version to be published, and agree to be accountable for all aspects of the work.

\section{Disclosure}

The authors report no conflicts of interest in this work.

\section{References}

1. Lee MS, Ahmad T, Lee J, et al. Dual delivery of growth factors with coacervate-coated poly(lactic-co-glycolic acid) nanofiber improves neovascularization in a mouse skin flap model. Biomaterials. 2017; 124:65-77.

2. Lin R, Lin J, Li S, et al. Effects of the traditional Chinese medicine baicalein on the viability of random pattern skin flaps in rats. Drug Des Devel Ther. 2018;12:2267-2276.

3. Fukunaga Y, Izawa-Ishizawa Y, Horinouchi Y, et al. Topical application of nitrosonifedipine, a novel radical scavenger, ameliorates ischemic skin flap necrosis in a mouse model. Wound Repair Regen. 2017;25(2): 217-223.

4. Chen T, Tu Q, Cheng L, Li Z, Lin D. Effects of curculigoside A on random skin flap survival in rats. Eur J Pharmacol. 2018;834:281-287.

5. Lin R, Chen H, Callow D, et al. Multifaceted effects of astragaloside IV on promotion of random pattern skin flap survival in rats. Am J Transl Res. 2017;9(9):4161-4172.

6. Yin Z, Ren H, Liu L, et al. Thioredoxin Protects Skin Flaps from Ischemia-Reperfusion Injury: A Novel Prognostic and Therapeutic Target. Plast Reconstr Surg. 2016;137(2):511-521.

7. Nanau RM, Neuman MG. Adverse drug reactions induced by valproic acid. Clin Biochem. 2013;46(15):1323-1338.

8. Chiu CT, Wang Z, Hunsberger JG, Chuang DM. Therapeutic potential of mood stabilizers lithium and valproic acid: beyond bipolar disorder. Pharmacol Rev. 2013;65(1):105-142.

9. Zhang L, Fan Z, Han Y, et al. Valproic Acid Promotes Survival of Facial Motor Neurons in Adult Rats After Facial Nerve Transection: a Pilot Study. J Mol Neurosci. 2018;64(4):512-522.

10. Wang Z, Tsai L-K, Munasinghe J, et al. Chronic Valproate Treatment Enhances Postischemic Angiogenesis and Promotes Functional Recovery in a Rat Model of Ischemic Stroke. Stroke. 2012;43(9):2430-2436.

11. Shao L, Young LT, Wang JF. Chronic treatment with mood stabilizers lithium and valproate prevents excitotoxicity by inhibiting oxidative stress in rat cerebral cortical cells. Biol Psychiatry. 2005;58(11): 879-884.

12. Michaelis M, Suhan T, Michaelis UR, et al. Valproic acid induces extracellular signal-regulated kinase $1 / 2$ activation and inhibits apoptosis in endothelial cells. Cell Death Differ. 2006;13(3):446-453.

13. Van Beneden K, Geers C, Pauwels M, et al. Valproic acid attenuates proteinuria and kidney injury. $J$ Am Soc Nephrol. 2011;22(10): $1863-1875$.

14. Zhou KL, Zhang YH, Lin DS, Tao XY, Xu HZ. Effects of calcitriol on random skin flap survival in rats. Sci Rep. 2016;6:18945.

15. Chen L, Zhou K, Chen H, Li S, Lin D, Zhou D. Calcitriol promotes survival of experimental random pattern flap via activation of autophagy. Am J Transl Res. 2017;9(8):3642-3653.

16. Kay HY, Greene DL, Kang S, Kosenko A, Hoshi N. M-current preservation contributes to anticonvulsant effects of valproic acid. J Clin Invest. 2015;125(10):3904-3914.

17. Fayazzadeh E, Yavarifar H, Rafie SR, Motamed S, Sotoudeh Anvari M, Boroumand MA. Fibroblast Growth Factor-1 vs. Fibroblast Growth Factor-2 in Ischemic Skin Flap Survival in a Rat Animal Model. World J Plast Surg. 2016;5(3):274-279.

18. Causey MW, Salgar S, Singh N, Martin M, Stallings JD. Valproic acid reversed pathologic endothelial cell gene expression profile associated with ischemia-reperfusion injury in a swine hemorrhagic shock model. J Vasc Surg. 2012;551103(4):1096-1103.e51. 
19. Yang Q, Tian Y, Liu S, et al. Thrombospondin-1 Peptide ABT-510 Combined with Valproic Acid Is an Effective Antiangiogenesis Strategy in Neuroblastoma. Cancer Res. 2007;67(4):1716-1724.

20. Yamanegi K, Kawabe M, Futani H, et al. Sodium valproate, a histone deacetylase inhibitor, modulates the vascular endothelial growth inhibitor-mediated cell death in human osteosarcoma and vascular endothelial cells. Int J Oncol. 2015;46(5):1994-2002.

21. Ying GY, Jing CH, Li JR, et al. Neuroprotective effects of valproic acid on blood-brain barrier disruption and apoptosis-related early brain injury in rats subjected to subarachnoid hemorrhage are modulated by heat shock protein 70/matrix metalloproteinases and heat shock protein 70/AKT pathways. Neurosurgery. 2016;79(2): 286-295.

22. Bousseau S, Vergori L, Soleti R, Lenaers G, Martinez MC, Andriantsitohaina R. Glycosylation as new pharmacological strategies for diseases associated with excessive angiogenesis. Pharmacol Ther. 2018;191:92-122.

23. Cao J, Ehling M, März S, et al. Polarized actin and VE-cadherin dynamics regulate junctional remodelling and cell migration during sprouting angiogenesis. Nat Commun. 2017;8(1):2210.

24. Cai L, Cao B, Lin D. Effects of Traditional Chinese Medicine Huangqi Injection (Radix astragali) on Random Skin Flap Survival in Rats. $J$ Reconstr Microsurg. 2015;31(08):565-570.

25. Afraz S, Kamran A, Moazzami K, Nezami BG, Dehpour AR. Protective effect of pharmacologic preconditioning with pioglitazone on randompattern skin flap in rat is mediated by nitric oxide system. J Surg Res. 2012;176(2):696-700.

26. Lin Y, Lin B, Lin D, Huang G, Cao B. Effect of Thymosin $\beta 4$ on the Survival of Random Skin Flaps in Rats. J Reconstr Microsurg. 2015; 31(6):464-470.

27. Kim EK, Hong JP. The effect of recombinant human erythropoietin on ischemia-reperfusion injury: an experimental study in a rat TRAM flap model. Plast Reconstr Surg. 2007;120(7):1774-1781.
28. Wang L, Ma Q. Clinical benefits and pharmacology of scutellarin: A comprehensive review. Pharmacol Ther. 2018;190:105-127.

29. Zweier J, Talukder M. The role of oxidants and free radicals in reperfusion injury. Cardiovasc Res. 2006;70(2):181-190.

30. Zaky A, Mahmoud M, Awad D, El Sabaa BM, Kandeel KM, Bassiouny AR. Valproic acid potentiates curcumin-mediated neuroprotection in lipopolysaccharide induced rats. Front Cell Neurosci. 2014; 8:337.

31. Yao Y, Miao W, Liu Z, et al. Dimethyl fumarate and monomethyl fumarate promote post-ischemic recovery in mice. Transl Stroke Res. 2016; 7(6):535-547.

32. Suda S, Ueda M, Nito C, et al. Valproic acid ameliorates ischemic brain injury in hyperglycemic rats with permanent middle cerebral occlusion. Brain Res. 2015;1606:1-8.

33. Zhao L, An R, Yang Y, et al. Melatonin alleviates brain injury in mice subjected to cecal ligation and puncture via attenuating inflammation, apoptosis, and oxidative stress: the role of SIRT1 signaling. $J$ Pineal Res. 2015;59(2):230-239.

34. Rah DK, Min HJ, Kim YW, Cheon YW. Effect of platelet-rich plasma on ischemia-reperfusion injury in a skin flap mouse model. Int J Med Sci. 2017;14(9):829-839.

35. Li Z, Wu F, Zhang X, et al. Valproate attenuates endoplasmic reticulum stress-induced apoptosis in SH-SY5Y cells via the AKT/GSK3 $\beta$ signaling pathway. Int J Mol Sci. 2017;18(2):E315.

36. Lee JY, Maeng S, Kang SR, et al. Valproic acid protects motor neuron death by inhibiting oxidative stress and endoplasmic reticulum stressmediated cytochrome $\mathrm{C}$ release after spinal cord injury. J Neurotrauma. 2014;31(6):582-594.

37. Johnson A, Francis M, DiPietro LA. Differential apoptosis in mucosal and dermal wound healing. Adv Wound Care (New Rochelle). 2014;3(12):751-761.

38. Cohen GM. Caspases: the executioners of apoptosis. Biochem J. 1997; 326(Pt 1):1-16.
Drug Design, Development and Therapy

\section{Publish your work in this journal}

Drug Design, Development and Therapy is an international, peerreviewed open-access journal that spans the spectrum of drug design and development through to clinical applications. Clinical outcomes, patient safety, and programs for the development and effective, safe, and sustained use of medicines are the features of the journal, which

\section{Dovepress}

has also been accepted for indexing on PubMed Central. The manuscript management system is completely online and includes a very quick and fair peer-review system, which is all easy to use. Visit http://www.dovepress.com/testimonials.php to read real quotes from published authors. 